\title{
The Risky Value in the Capital Market of Incomplete Information
}

\author{
Xuhong Tan \& Mingjun Tan \\ Department of Economics\& Management, Heilongjiang Institution of Science and Technology \\ Harbin 150027, China
}

\begin{abstract}
Risk is everywhere, especially in the capital market of incomplete information, which is full of speculation, so it is particularly important for investors to define risk, identify risks, and control the investment activities with the application of risk value to. It is analyzed quantitatively on risk value in the paper, with the Knowledge of game theory, economics and mathematical statistics, on the basis of the explanation of risk and risk value.
\end{abstract}

Keywords: Risky value, Incomplete information, Risk Aversion, Investment Portfolio

\section{Introduction}

What is risk? Different scholars have different interpretations. Some people think that risk is a kind of uncertainty, including the uncertainty whether the incident may be determined or the Probability of the occurrence; while some people think that risk is a concern for future events, including some doubts on the outcome of the future and some doubts on the possibility of the objectively existed damage. Although these explanations are true in certain degree, it seems not be exact to express the real content of risk. We think that risk is kinds of uncertainties of various results in certain period and certain conditions. This definition includes two Connotations: (1) risk is the uncertainty of the event which is objective. Specifically, it means various results under uncertain conditions, and the possibility of the occurrence. Therefore, risk is objective, but whether people are willing to and how to take risks is subjective. Strictly speaking, uncertainty and risk exists some differences, risk refers the results and the probability of events, which is measurable; while uncertainty is immeasurable, which means it's impossible to predict the results and the probability of various outcomes. (2) the degree of the risk is change with the passage of time. That is, Specific risk exists in a certain period of time, during which, as time goes by, the uncertainty of events is diminishing, and when it's completed at the end; the results can be fully identified. Therefore, it is necessary to adjust the early predictive number in accordance with the progress in the process of risk prediction. In the angle of mathematical statistics, the mathematical models of risk can be expressed as:

$$
\sigma=\sqrt{\sum_{i=1}^{n}\left(x_{i}-\bar{x}\right)^{2} \cdot p_{i}} \quad \text { OR } \quad v=\frac{\sigma}{\bar{x}}
$$

Where, $\sigma$ denotes standard deviation; $x_{i}$ denotes earnings of investment; $\bar{x}$ denotes anticipated earnings; $p_{i}$ denotes the Probabilities of events; $v$ denotes the ratio of standard deviation.

On the basis of definition on the concept of risk, we are now to discuss risky value. The so-called risky value is the excessive earnings requested by investors, which is more than the present value of investment funds. Generally speaking, the greater the risk, the more he returns requested, vice versa, following is the model:

$$
k=k_{0}+k_{R}=k_{0}+b v
$$

Where, $k$ denotes the rate of return requested by the investors; $k_{0}$ denotes the rate of risk-free return; $k_{R}$ denotes the rate of risky return; $b$ denotes the coefficient of risky value; $v$ denotes the rate of standard deviation.

\section{Incomplete information and risky value}

The so-called incomplete information means that the market participant is not able to take full possession of the all knowledge or information in the certain economic environment, which is particularly obvious in the initial stage of the imperfect market. According to the principle of effective capital market, the present value of the investment to any financial instrument is zero, that is, it is impossible for any investor to increase shareholders' value by trading of financial assets in the capital markets, and it's impossible to obtain excess earnings for any speculation. However, in the view of new Keynesian, incomplete information is more economic reality than complete information. The presence of incomplete information will lead to malfunctioning of the market price mechanism. In the capital markets ,trading parties take investment transactions on the principles of returns maximization, but the imperfection of the market and incomplete information will lead the market failures, and more importantly, the dissemination of information is usually in large cost, as well, the limitations of market communications systems and the objective and 
subjective factors such as market participants release the market noise, will also seriously hamper the exchange of information and effective communication. As previously mentioned, the results is that the price information can not be timely passed to each market participant who needs the information, and trading activities and its results can not be promptly transmitted through the price system, Therefore, the market prices cannot reflect sensitively the supply and demand in the market, which, makes the price of certain financial assets far away from its intrinsic value, resulting in the formation of various speculative.

\section{The application of risky value}

In the capital market of incomplete information, it is an important subject on how to avert risk in risk investment and risk management. The so-called risk aversion refers how to minimize risk the in certain income or how to maximize the income at certain risk. The most commonly used measure is investment portfolio (it's also named scattered effect), the method takes an important role in decentralizing the non-systemic risk. Under the conditions of the investment portfolio, the risk models:

$$
\begin{aligned}
& \sigma=\sqrt{\sum_{i=1}^{m} \sum_{j=1}^{m} w_{i} w_{j} r_{i j} \sigma_{i} \sigma_{j} \quad(m \geq 2)} \\
& r_{i j}=\frac{\sum\left[\left(x_{i}-\bar{x}_{i}\right)\left(x_{j}-\bar{x}_{j}\right)\right]}{\sqrt{\sum\left(x_{i}-\bar{x}_{i}\right)^{2}} \sqrt{\sum\left(x_{j}-\bar{x}_{j}\right)^{2}}}
\end{aligned}
$$

Where, $m$ denotes the types of investment portfolio; $w_{i}$ and $w_{j}$ denotes the weights of the investment $i$ and $\mathrm{j}$; $\sigma_{i}$ and $\sigma_{j}$ denotes the standard deviation of the investment $i$ and $\mathrm{j} ; r_{i j}$ denotes the correlation coefficient of the investment $i$ and $\mathrm{j}$.

Following is the specification to model (3.1):

(1) when $\mathrm{m}=2$, then $\sigma=\sqrt{w_{1}^{2} \sigma_{1}^{2}+w_{2}^{2} \sigma_{2}^{2}+2 w_{1} w_{2} \sigma_{1} \sigma_{2} \cdot \operatorname{Corr}\left(x_{1}, x_{2}\right)}$

Where, $\operatorname{Corr}\left(x_{1}, x_{2}\right)$ denotes correlation coefficient.

(2) when $m>2$, that is, exists a variety of asset portfolio, the following is to prove the feasibility of dispersion effects from the perspective of game theory:

Located exist $m$ combination of options, for which the corresponding income is: $x_{1}, x_{2}, \ldots x_{m}$, and its distribution is independent, then there is a viable strategy vector $\mathrm{C}=\left\{w_{1}, w_{2}, \ldots w_{m}\right\}$, in which, $w_{i}$ is the weights in the portfolio $i$, and $\sum_{i=1}^{m} w_{1}=1$, then its net payment $\tilde{y}=\sum_{i=1}^{m} w_{1} x_{i}$. Now consider any viable alternative strategies for:

$$
\sum_{i=1}^{m} w_{i} x_{i}=\tilde{y}+\sum_{i=1}^{m}\left(w_{i}-\frac{1}{m}\right) x_{i}
$$

If the conditions mean for $\sum_{i=1}^{m}\left(w_{1}-\frac{1}{m}\right) x_{i}$ is zero, then:

$$
E\left[\sum_{i=1}^{m}\left(w_{i}-\frac{1}{m}\right) x_{i} \mid \tilde{y}\right]=\sum_{i=1}^{m}\left(w_{i}-\frac{1}{m}\right) E\left[x_{i} \mid \tilde{y}\right]
$$

According to symmetry, $E\left[x_{i} \mid \tilde{y}\right]$ is independent to $i$, then:

$$
E\left[\sum_{i=1}^{m}\left(w_{i}-\frac{1}{m}\right) x_{i} \mid \tilde{y}\right]=c \sum_{i=1}^{m}\left(w_{i}-\frac{1}{m}\right)=0
$$

It's proved that alternative strategies were second-order random dominant to completely decentralized strategy, which means that all the parties who are risk-averters will choose completely decentralized strategy. And when $n \rightarrow \infty$, it is allowed to replace the unit risky earning $x_{i}$ with its expected value in the strategy, and that is an application of large number theorem.

\section{Conclusion}

In the capital market of incomplete information, particularly in China's capital market which is in a special period of economy transformation, it is the most important aspect in China's socialist market economy that how to regulate the operational mechanisms of the capital market, and how to establish the mature capital market and improve the security mechanism of risk investment. Of course, we have reasons to believe that, as the healthy and stable 
development of china's economy and the continuous self-improvement of China's capital market, risk investment in China will be more developed, and investors' awareness of the risk management will become increasingly strong, investment behavior will more rational, as well.

\section{References}

Jewitt. (1965). Risk aversion and the choice between risky prospcts: the preservation of comparative staticts. Review of economic studies. 54:73-85.

Jowett Paul \& Margaret. Rothwell. (1986). The economics of information technology. st. martin's press.

Kimball. (1972). Standard risk aversion. Econometric. 61:589-611. 